\title{
Un exemple d'étude rhéophysique(l): L'électrorotation appliquée au contrôle de la viscosité d'une suspension
}

Élisabeth Lemaire (elisabeth.lemaire@unice.fr),

Laurent Lobry, Nicolas Pannacci et François Peters

LPMC, CNRS-Université de Nice, 06108 Nice Cedex 2

La rotation de Quincke décrit la mise en rotation d'objets par un champ électrique continu. Nous montrons ici comment nous avons pu abaisser la viscosité apparente d'une suspension de près d'un ordre de grandeur en la soumettant à un tel champ électrique.

(1) La rhéophysique est une discipline née dans les années 80 , qui s'efforce de prédire ou tout au moins d'expliquer les propriétés mécaniques d'un système à l'aide d'arguments physiques. $\mathrm{La}$ démarche généralement adoptée est d'étudier les interactions et/ou la structure d'un matériau complexe à l'échelle de ses constituants, et d'en déduire ses propriétés macroscopiques moyennes.

Figure 1 : Principe de la mise en rotation d'une particule sous l'effet d'un champ électrique continu. Si le temps de relaxation des charges est plus faible dans le liquide que dans la particule, sous l'effet du champ électrique, la particule acquiert une distribution surfacique de charges équivalente à celle d'un dipôle en sens inverse du champ appliqué (a). Si l'intensité du champ est assez grande, la particule se met en rotation pour tenter d'aligner le dipôle P dans la direction du champ (b).
Lorsqu'une particule isolante, immergée dans un liquide légèrement conducteur, est soumise à un champ électrique continu suffisamment grand, elle se met spontanément en rotation sur elle-même autour d'un axe perpendiculaire au champ appliqué. Bien que la première observation de ce phénomène, appelé « rotation de Quincke ", soit très ancienne (le premier article qui en fait mention date de 1896), il demeure assez peu connu ; il a fallu, par exemple, attendre près d'un siècle pour qu'une interprétation quantitative en soit donnée [1].

Aujourd'hui encore, la rotation de Quincke nous réserve de belles surprises, qu'il s'agisse du comportement d'une particule isolée ou d'une assemblée de particules. Nous avons, par exemple, montré que la dynamique du rotor de Quincke dans sa géométrie classique - un cylindre libre de tourner autour de son axe placé perpendiculairement à un champ électrique - était régie par les célèbres équations de Lorenz sans aucune approximation [2]. Lorsqu'on se souvient du rôle tout à fait important et précurseur qu'ont joué ces équations dans la compréhension du chaos déterministe [3], on mesure l'intérêt de disposer d'un système expérimental leur obéissant exactement. La rotation de Quincke a aussi des conséquences inattendues sur l'orientation de fibres diélectriques soumises à un champ électrique continu. Elle permet, en particulier, de stabiliser une orientation

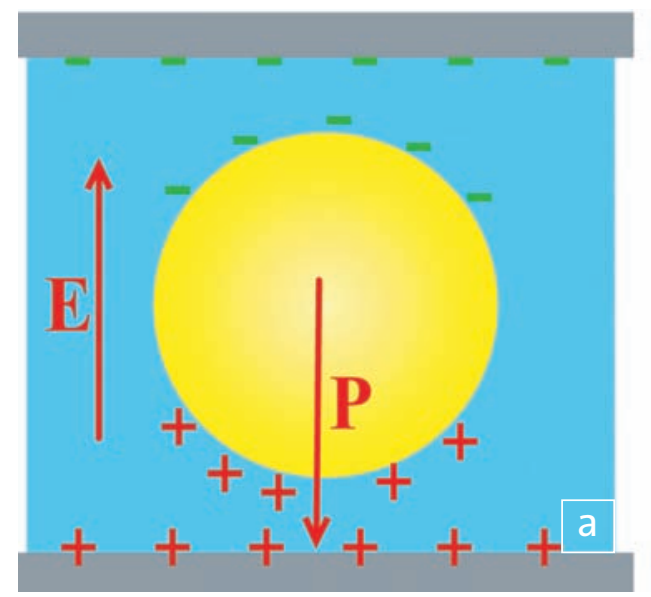

des fibres perpendiculaire au champ [4]. Lorsqu'on s'intéresse au comportement collectif d'une assemblée de particules suspendues dans un liquide, on s'aperçoit que la rotation de Quincke peut conduire à une augmentation notable de la conductivité électrique effective de la suspension [5]. Nous allons ici discuter de l'utilisation de la rotation de Quincke pour abaisser la viscosité apparente d'une suspension.

\section{Électrorotation sous champ} continu : la rotation de Quincke

Auparavant, décrivons le mécanisme responsable de la mise en rotation des particules et examinons l'action des charges libres présentes dans le liquide. Lorsqu'un champ électrique continu $\mathbf{E}$ est appliqué, les ions migrent d'une électrode vers l'autre et, rencontrant la particule isolante, s'accumulent à sa surface en induisant un dipôle, $\mathbf{P}$, dans la direction opposée au champ électrique (fig. 1a). Pour un champ électrique d'intensité suffisamment grande, cet équilibre est instable et la particule se met en rotation pour tenter d'orienter son dipôle dans la direction du champ appliqué. En tournant, elle entraîne ses charges superficielles mais, dans le même temps, de nouvelles charges arrivent à sa surface. La particule ne réussit donc pas à aligner son dipôle dans la direction du champ (fig. 1b). Par conséquent, en régime stationnaire, le dipôle

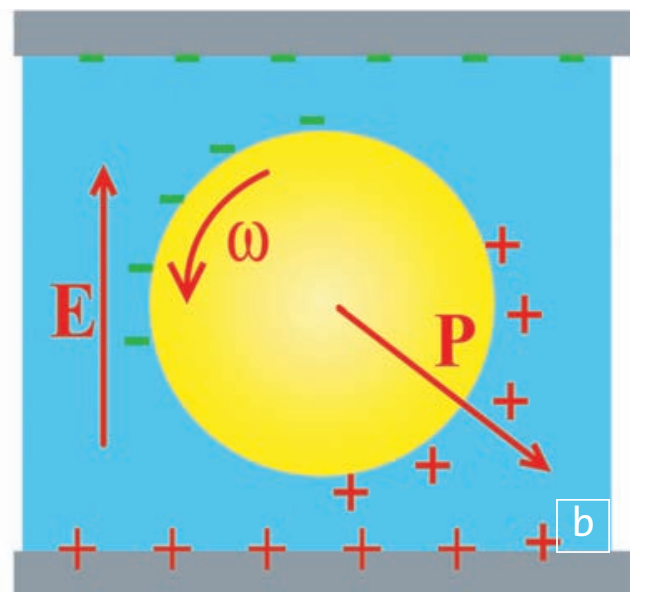


Figure 2 : Variation de l'opposé du couple hydrodynamique exercé par le fluide sur la particule (courbe noire) et du couple électrique (courbes bleue et rouge) qu'elle subit pour deux intensités du champ électrique, en fonction de la vitesse angulaire normalisée par l'inverse du temps de Maxwell. II apparait clairement que si l'intensité du champ électrique n'atteint pas une valeur seuil, la particule ne se met pas en rotation (courbe en bleu).

Figure 3 : Quand une particule placée entre deux plaques tourne sur elle-même autour d'un axe parallèle aux plaques, elle crée un champ de vitesse dans le fluide qui exerce alors une force sur les plaques.

(2) Le champ seuil de mise en rotation d'une particule est d'autant plus grand que la viscosité et la conductivité électrique du liquide sont élevées. Ainsi, puisqu'on ne peut pas appliquer de champ électrique aussi grand que l'on veut, en particulier en raison des claquages diélectriques qui surviennent au-delà d'une intensité critique, les fluides utilisés ne devront pas être "trop " conducteurs. Cette limitation nous interdira, par exemple, de travailler en milieu aqueux et nous conduira à utiliser des fluides peu polaires (de faible constante diélectrique), dont les conductivités seront typiquement comprises entre $10^{-9}$ et $10^{-7} \mathrm{~S} / \mathrm{m}$. Les fluides polaires sont généralement des huiles ou des hydrocarbures dont la viscosité est de l'ordre de $10 \mathrm{mPa} . \mathrm{s}$ (10 fois celle de l'eau). Dans ces conditions, les champs seuil sont compris entre 100 et $1000 \mathrm{~V} / \mathrm{mm}$ et les vitesses de rotation inférieures à 10 fois l'inverse du temps de Maxwell, soit quelques milliers de radians par seconde.

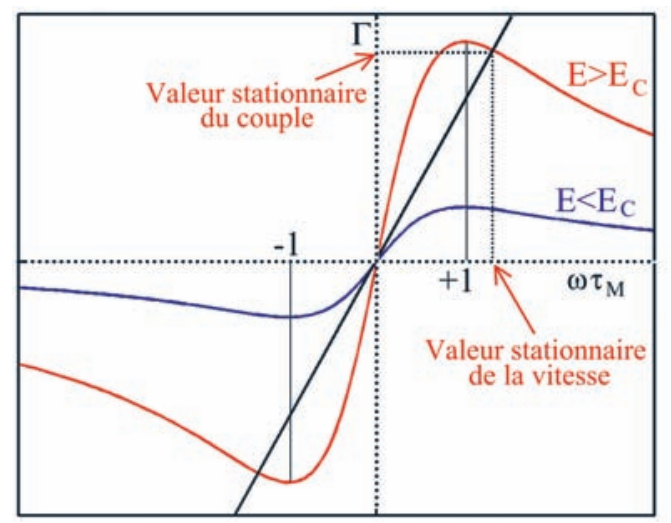

forme un angle constant non nul avec le champ électrique et la particule subit un couple moteur, $\Gamma \mathbf{E}=\mathbf{P} \mathbf{X E}$, qui est équilibré par le couple résistant hydrodynamique, $\Gamma^{\mathbf{H}}=-\alpha \omega$, où $\omega$ est la vitesse angulaire de la particule, et $\alpha$ son coefficient de friction rotationnel. Notons qu'en régime stationnaire, l'orientation du dipôle et la vitesse de rotation sont indépendantes de la taille de la particule, car $\alpha$ et $\mathbf{P}$ dépendent tous deux linéairement du volume de la particule. Précisons, par ailleurs, que le dipôle associé à la charge surfacique de la particule dépend de sa vitesse de rotation ; il est déterminé par une équation de relaxation traduisant que sa variation temporelle est donnée par la somme de deux termes [5] :

- un terme d'entraînement du dipôle (i.e. de la charge de surface) par la rotation de la particule,

- un terme de relaxation du dipôle vers sa valeur d'équilibre en absence de rotation avec un temps caractéristique $\tau_{\mathrm{M}}$, le temps de Maxwell. La relaxation du dipôle correspond évidemment à la relaxation des charges de surface. Il existe donc un lien étroit entre le temps de Maxwell et le temps de relaxation de la charge classiquement observé dans un milieu conducteur. Celui-ci est donné par le rapport de la permittivité diélectrique sur la conductivité. Pour les systèmes qui nous intéressent, il est de l'ordre de quelques millisecondes.

La figure 2, qui représente la variation des couples électrique et visqueux en fonction de la vitesse de rotation de la particule, appelle plusieurs commentaires. Remarquons d'abord qu'il existe un champ électrique, $\mathrm{E}_{\mathrm{c}}$, en dessous duquel la particule reste immobile, car quelle que soit la vitesse de rotation de la particule, le couple hydrodynamique résistant est supérieur au couple électrique moteur. Lorsqu'elle est soumise à un champ d'intensité supérieure à la valeur seuil, la particule se met en rotation à la vitesse $\pm \omega$. En réalité, si la particule est sphérique, elle pourra se mettre en rotation autour de n'importe quel axe perpendiculaire au champ électrique et ce sont les conditions initiales qui détermineront la direction de la rotation.
La variation de la vitesse angulaire en fonction du champ électrique obéit à une loi très simple, où il apparait que l'ordre de grandeur typique de la vitesse de rotation est donné par l'inverse du temps de Maxwell, soit quelques centaines de radians par seconde(2) :

$$
\omega=\left(\tau_{M}\right)^{-1}\left[\left(E / E_{c}\right)^{2}-1\right]^{1 / 2}
$$

Cet ordre de grandeur est également celui de la vitesse de rotation des particules d'une suspension soumise à un écoulement de cisaillement simple dans une expérience typique de rhéologie. La rotation de Quincke devrait ainsi avoir une influence notable sur le comportement rhéologique (mécanique) d'une suspension. Nous verrons dans la suite que cette idée est réaliste, et comment nous avons pu obtenir une baisse de viscosité apparente d'un ordre de grandeur ou plus.

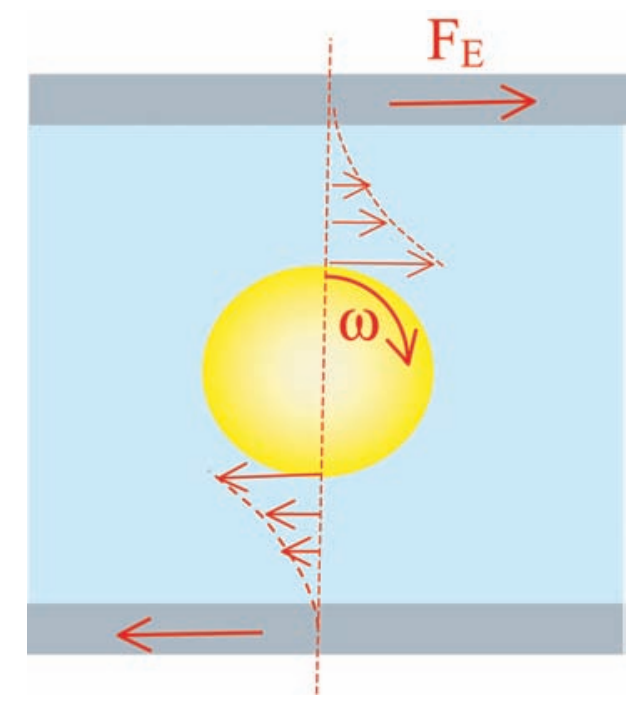

\section{Rotation des particules et viscosité apparente d'une suspension}

Présentons en premier lieu le mécanisme permettant d'expliquer la baisse de viscosité lorsque les particules sont soumises à un couple. Imaginons pour cela une particule immergée dans un liquide et placée entre deux plaques (fig. 3).Lorsque la particule tourne sur elle-même autour d'un axe parallèle aux plaques sous l'action d'un champ électrique, elle entraîne le liquide qui l'entoure, puisque celui-ci est visqueux. De la sorte, il apparaît dans le liquide un champ de vitesse qui tend à mettre en mouvement les plaques, chacune dans un sens différent. Ainsi, le couple électrique qui met en rotation la particule est à l'origine d'une force visqueuse, $\mathbf{F}_{\mathbf{E}}$, sur les plaques. Examinons maintenant l'effet du champ électrique sur le comportement rhéologique d'une suspension qu'on imagine placée entre deux plans parallèles. De façon générale, la viscosité d'un fluide peut être déterminée à 

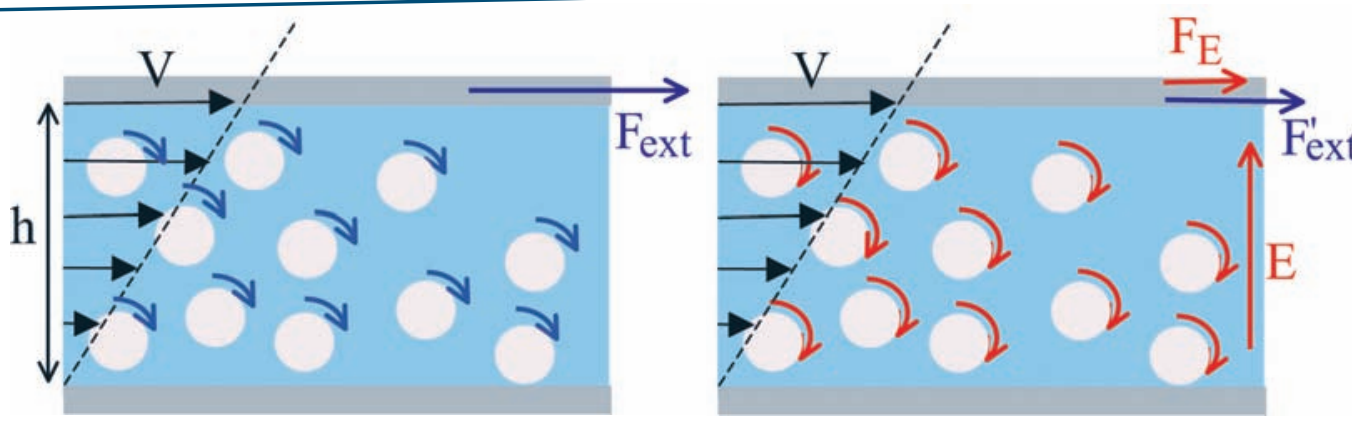

l'aide d'une expérience élémentaire consistant à maintenir l'un des deux plans immobile, tandis que l'on tire sur l'autre à force constante, $\mathbf{F}_{\text {ext }}$ (fig. 4a) [6]. Lors de cette expérience de cisaillement, un gradient de vitesse constant, $\mathbf{V} /$ h, apparait dans la suspension. En faisant le rapport de la contrainte de cisaillement $\left(\boldsymbol{\sigma}=\mathbf{F}_{\text {ext }} / \mathrm{S}\right)$ sur le gradient de vitesse (ou taux de cisaillement), on déduit directement la viscosité effective de la suspension.

Ainsi, lorsque le champ électrique est appliqué, la force $\mathbf{F}_{\mathbf{E}}$ vient s'ajouter à la force exercée par l'opérateur sur le plan mobile et, si l'on veut que ce dernier continue de se déplacer à la vitesse $\mathbf{V}$, il faudra appliquer une force $\mathbf{F}^{\prime}{ }_{\text {ext }}$ plus faible qu'en absence de champ. La viscosité apparente aura donc décru (fig. 4b).

La figure 5 présente le comportement rhéologique d'une suspension de particules de PMMA dispersées dans un mélange de deux liquides diélectriques (Dielec S et Ugilec), réalisé dans le but d'ajuster la densité du fluide suspendant à celle des particules. À l'état pur, ce mélange est très peu conducteur ; nous avons ajouté des sels d'AOT (dodecyl sulfosuccinate de sodium) afin d'amener la conductivité à une valeur de l'ordre de $10^{-8} \mathrm{~S} / \mathrm{m}$. La baisse de viscosité enregistrée, lorsqu'un champ électrique de $2 \mathrm{kV} / \mathrm{mm}$ est appliqué, est considérable (un facteur 5). En particulier, pour un champ suffisamment grand, la viscosité apparente de la suspension devient bien inférieure à celle du liquide suspendant, dont la valeur est de $16 \mathrm{mPa}$.s, montrant sans conteste le rôle de micromoteurs joué par les particules soumises à la rotation de Quincke.
Les traits de couleur représentent les prédictions théoriques que nous avons pu faire en utilisant un modèle simple basé sur les hypothèses énoncées ci-dessus. L'accord avec les résultats expérimentaux est assez bon, sauf en ce qui concerne les faibles taux de cisaillement et les plus petits champs pour lesquels, contrairement à ce que nous avions prédit, les expériences montrent une augmentation de viscosité. Cette augmentation de la viscosité de la suspension provient très probablement des interactions dipolaires entre particules, qui ne sont pas considérées dans notre modèle et qui conduisent à une structuration de la suspension en feuillets parallèles à la direction de l'écoulement et du champ électrique [7].

\section{Conclusion}

La rotation de Quincke constitue ainsi un exemple d'un sujet d'étude ancien qui, appliqué au domaine des fluides complexes, soulève des problèmes de physique contemporaine. Se posent en effet des questions fondamentales de rhéophysique concernant les liens entre les propriétés mécaniques macroscopiques d'une suspension et la dynamique de ses constituants microscopiques. D'autre part, il semble que l'ampleur des variations de viscosité induites par la rotation de Quincke, alliée à la simplicité de mise en œuvre du contrôle (champ électrique continu) et à la robustesse physico-chimique du matériau, donnent à ces fluides adaptatifs un potentiel d'applications important dans le domaine du visco-couplage ou de l'amortissement actif de vibrations.

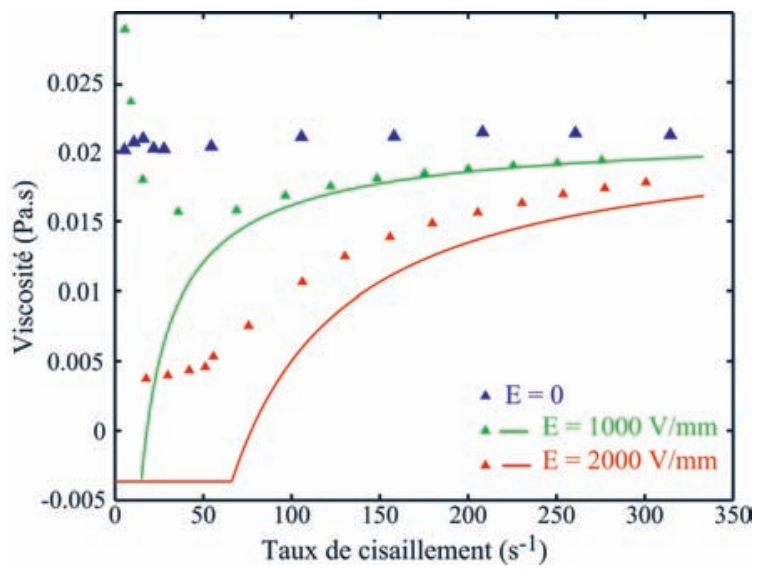

Figure 5 : Viscosité apparente d'une suspension de particules de PMMA (diamètre $70 \mu \mathrm{m}$, fraction volumique $10 \%$, permittivité $2,6 \varepsilon_{0}$, conductivité $10^{-14} \mathrm{~S} / \mathrm{m}$ ) dispersées dans un fluide diélectrique (permittivité $3,7 \varepsilon_{0}$, conductivité 1,45 10-8 $\mathrm{S} / \mathrm{m}$ ). Les symboles correspondent aux mesures expérimentales, tandis que les lignes représentent les prédictions du modèle. L'application d'un champ électrique a pour effet d'abaisser la viscosité de la suspension jusqu'à des valeurs très inférieures à celles de la viscosité du fluide suspendant. Pour les taux de cisaillement les plus élevés, l'augmentation de vitesse de rotation des particules induite par le champ tend a devenir négligeable devant leur vitesse induite par le cisaillement. La viscosité de la suspension tend alors vers sa valeur en absence de champ électrique.
Figure 4 : Lorsqu'une suspension est soumise à un écoulement de cisaillement simple, on peut montrer que les particules qui la composent tournent sur elles-mêmes à une vitesse égale à la moitié du gradient de vitesse. Si on augmente la vitesse de rotation des particules en appliquant un champ électrique dans la direction du gradient de vitesse, la rotation des particules facilite l'écoulement du fluide et la viscosité apparente de la suspension diminue.

\section{Références}

[1] T.B. Jones, IEEE Trans. on Industry Applications, IA-20 (1984) 845.

[2] F. Peters, L. Lobry, E. Lemaire, "Experimental observation of Lorenz chaos in the Quincke rotor dynamics", Chaos 15 (2005) 013102.

[3] P. Bergé,Y. Pomeau, C.Vidal, L'ordre dans le chaos, collection

"Enseignement des sciences",

Hermann, Paris (1998).

[4] A. Cebers, L. Lobry, E. Lemaire, "Electrohydrodynamic instabilities and orientation of dielectric

ellipsoids in low conducting fluids", Phys. Rev. E 63 (2001) 016301.

[5] A. Cebers, L. Lobry, E. Lemaire, "Internal rotations in dielectric suspensions", Magnitnaya Gidrodinamica 36 (2000) 347.

[6] G. Couarraze, J.L. Grossiord, Initiation à la rhéologie,

collection «Technique et documentation ", Lavoisier (1983).

[7] N. Pannacci, E. Lemaire,

L. Lobry, "Rheology and structure of a suspension of particles subjected to Quincke rotation", Rheol. Acta 46 (2007) 899-904 